\title{
Effect of Spinal Cord Stimulation for Patients with Post-Zoster Neuralgia on the Trunk: A Case Report
}

\author{
Jong-Ho Ahn, Seu-Ryang Jang, Sang-Bok Lee, Young-Woo Kim, Tae-Kyu Lee ${ }^{\bowtie}$ \\ Department of Neurosurgery, Uijeongbu St. Mary's Hospital, College of Medicine, The Catholic University of Korea, Uijeongbu, Korea
}

\begin{abstract}
Post-zoster neuralgia is an intractable pain by medical treatment. However, practice guidelines for invasive procedures of medically intractable post-zoster neuralgia are unclear, and it is not clear to apply to individual cases. In our study, the effect of spinal cord stimulation (SCS) for patients with post-zoster neuralgia was described in old patients with episodic pain with constant pain, which have been poorly controlled with oral and invasive treatments. We used an SCS device (Synergy2 IPG, model 7427; Medtronic Inc, Minneapolis, MN) equipped with an electrode (1×8 standard Lead model 977A290; Medtronic Inc, Minneapolis, MN). One lead was placed in the left lower margin of the T12 vertebral body (pulse width $60 \mathrm{~ms}$, pulse rate $20 \mathrm{~Hz}$, amplitude $2.5 \mathrm{~V}$ ) and it covered on the distribution to left $10^{\text {th }}, 11^{\text {th }}, 12^{\text {th }}$ thoracic dermatomes. VAS decreased to 3-4 after SCS compared with baseline VAS and was sustained by 3-4 for 1 week and by 3-4 at 1 month. After 6 months, the pain remained at 4-5. In morphine consumption, the baseline doses were $200 \mathrm{mg} /$ day that significantly reduced to $120 \mathrm{mg} /$ day and $90 \mathrm{mg} /$ day after 1 -month and 6-months post-procedure. The dose of gabapentin $(1,800 \mathrm{mg} /$ day) before treatment. The dose of gabapentin in the first month did not significantly decrease in taking gabapentin $(1,200 \mathrm{mg} /$ day $)$ compared with the baseline dose $(1,200 \mathrm{mg} /$ day). Consumption after 6 months was also significantly reduced by $900 \mathrm{mg} /$ day.
\end{abstract}

Key Words: Post-zoster neuralgia; Postherpetic neuralgia, Pulsed radiofrequency; Spinal cord stimulation

$\triangle$ Corresponding Author: Tae-Kyu Lee, Department of Neurosurgery, Uijeongbu St. Mary's Hospital, College of Medicine, The Catholic University of Korea, 271, Cheon Bo-Ro, Uijeongbu 11765, Korea. Tel: +82-31-820-3638, Fax: +82-31-847-8077, E-mail: magpie67@catholic.ac.kr

\section{INTRODUCTION}

A small number of patients experience pain during 4 weeks of herpes zoster infection, and post-zoster neuralgia occurs in $10 \%$ of the patient after herpes zoster ${ }^{2}$. The combination therapy of pharmacologic agents is more effective than any monotherapy for the management of post-zoster neuralgia ${ }^{4)}$. However, invasive practical guidelines for medically intractable patients failing current drug therapy are unclear, and it is not clear to apply to the individual instructions ${ }^{3)}$. Therefore, the objective of this report is to evaluate the efficacy of spinal cord stimulation from a case with post-zoster neuralgia on left $10^{\text {th }}, 11^{\text {th }}, 12^{\text {th }}$ thoracic dermatomes

\section{CASE REPORT}

A 68-year-old male had a history of healing in herpes zoster infection with rash and blister on left $10^{\text {th }}, 11^{\text {th }}, 12^{\text {th }}$ thoracic dermatomes. This patient with severe left trunk pain for 126 months was referred to our pain clinic for adjustable pain management. Post-zoster neuralgia began following bullae and cluster in a herpes zoster infection on the distribution to left $10^{\text {th }}, 11^{\text {th }}, 12^{\text {th }}$ thoracic dermatomes. He described the pain as itching and lancinating sensation with constant and severe (VAS 7 to 9 as being the most severe). The patient did not respond to various drug treatments and combination treatment for relief of trunk pain: IRcodon $60 \mathrm{mg}$ bid, acetaminophen/hydrocodone $5 / 500$ tid, pregabalin $150 \mathrm{mg}$ bid, topical agents, the combination treatment of injection to scar and ICNB. For the relief of his post-zoster neuralgia, We used an SCS device (Synergy2 IPG, model 7427; Medtronic Inc, Minneapolis, MN) equipped with an electrode $(1 \times 8$ standard Lead model 977A290; Medtronic Inc, Minneapolis, MN). One lead was placed in the left lower margin of the T12 vertebral body (pulse width $60 \mathrm{~ms}$, pulse rate $20 \mathrm{~Hz}$, amplitude $2.5 \mathrm{~V}$ ), and is covered on the distribution to left $10^{\text {th }}, 11^{\text {th }}, 12^{\text {th }}$ thoracic dermatomes (Figs. 1, 2). After the trial stimulation, his visual analog score (VAS) decreased from 9 to 4. SCS-induced paresthesia covered his painful areas fully enough. The VAS of baseline status was 7-9 before SCS in the management of episodic pain with constant pain in patients with post-zoster neuralgia. VAS decreased to 3-4 after SCS compared with baseline VAS and was sustained by 3-4 for 1 week and by 3-4 at 1 month. After 6 months, the pain remained at 4-5. In morphine consumption, the baseline doses 

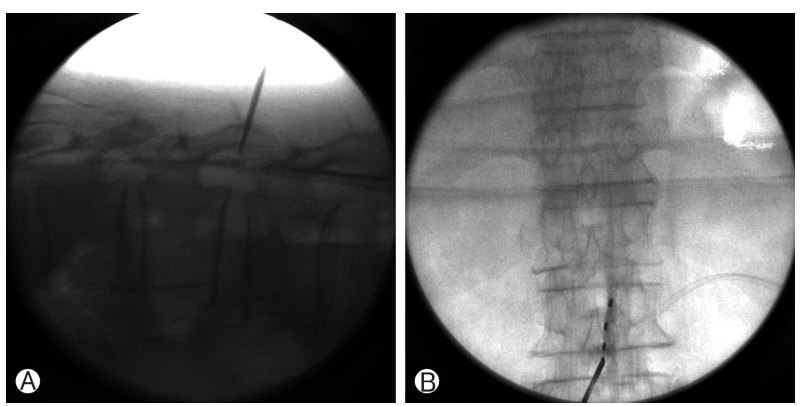

Fig. 1. (A) The root space of thoracic spine was accessed at L2-3 interspace and the C-arm rotated until the rootlet space was easily identified. (B) A 14-gauge, 10-cm Tuohy needle was advanced under fluoroscopic guidance with a direct approach.
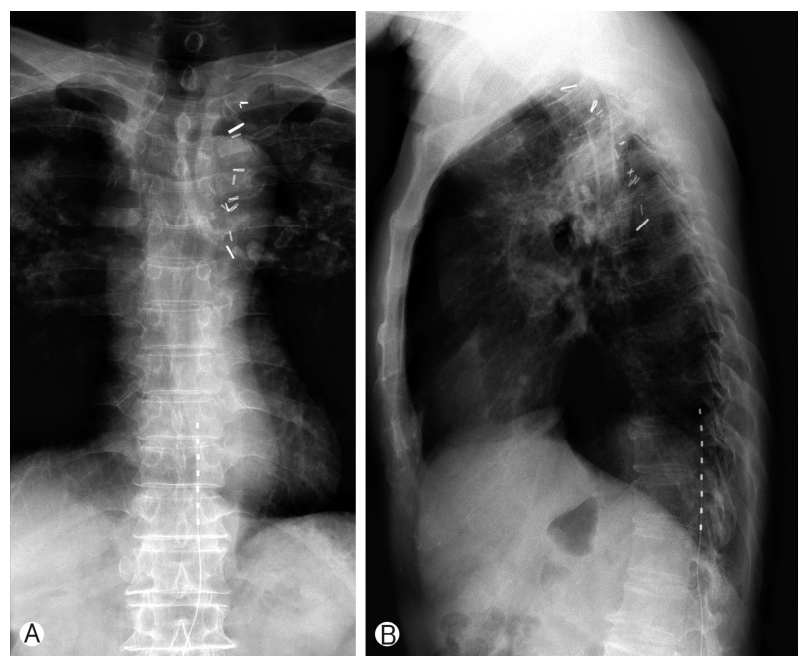

Fig. 2. An electrode ( $1 \times 8$ standard Lead model 977A290; Medtronic Inc, Minneapolis, MN) was placed in the left lower margin of the T12 vertebral body an electrode ( $1 \times 8$ standard Lead model 977A290; Medtronic Inc, Minneapolis, MN) (A: D-spine AP view, B: D-spine lateral view).

were $200 \mathrm{mg} /$ day that significantly reduced to $120 \mathrm{mg} /$ day and $90 \mathrm{mg}$ /day after one 1-month and 6-months post-procedure. The dose of gabapentin $(1,800 \mathrm{mg} /$ day $)$ before treatment. The dose of gabapentin in the first month did not significantly decrease in taking gabapentin $(1,200 \mathrm{mg} /$ day $)$ compared with the baseline dose (1,200 mg/day). Consumption after 6 months was also significantly reduced by $900 \mathrm{mg} /$ day.

\section{DISCUSSION}

During the acute herpes zoster infection, damage of peripheral nerves lowered threshold for pain activation from nociceptive organ and spontaneously ectopic discharges by destructed nerve cause peripheral and central sensitization ${ }^{4}$. Neurons of dorsal horn in spinal cord are also altered by response to the activity and inflammatory changes in peripheral nerves impaired descending inhibition of pain sensation and led to central sensitization $^{6,10)}$.

Treatment guidelines of post-zoster neuralgia suggested that medical treatment in post-zoster neuralgia is excellent in an effect of combination therapy of pharmacologic agents rather than a single therapy ${ }^{5}$. Looking at the drug treatment guidelines for post-zoster neuralgia issued by the International Association for the Study of Pain (IASP), First-line treatments include TCAs (tricyclic antidepressants), pregabalin and gabapentin, and topical lidocaine, 5\% opioid patch, capsaicin cream, and 8\% capsaicin patch are additionally recommended. Traditionally, anticonvulsants as carbamazepine and valproic acid showed an effect in some patients, and botulinum toxin had been applied to pain management. SSRIs (Selective serotonin reuptake inhibitors) and SNRIs (serotonin-norepinephrine reuptake inhibitors) have been applied to the medical treatment of post-zoster neuralgia $^{9,11)}$.

In comparison, the subcutaneous injection of lidocaine and triamcinolone is a safe and straightforward method to block affected peripheral nerves and reduce inflammation. In the literature, the pain NRS scores decreased significantly in all patients with post-zoster neuralgia one month after rash onset. Still, patients who received standard treatment plus subcutaneous injection showed substantially more significant improvement in pain scores than those who received standard therapy alone $^{1)}$.

Some presented 2 subacute PHN cases within 3 months of the onset of herpes zoster, in which limited duration SCS was effective in alleviating the pain. Increasing drug dosages or switching drugs was difficult because both patients were elderly ${ }^{8}$.

The use of SCS within 0.5 to 2 months from the onset of herpes zoster improved VAS scores to zero, and the patients could complete the therapies in 2.5 months. Responses in post-zoster neuralgia patients were not uniform; however, as some patients still had pain after 29 months of the treatment ${ }^{7)}$. Some proposed to utilize SCS for approximately 2 weeks without permanent implantation of the pulse generator. They showed that patients who were within 3 months from the onset of herpes zoster achieved pain relief ${ }^{12)}$. Some reported that early intervention with SCS provided better pain relief as well as longer periods of effective control. Also, long-term pain alleviation and patient satisfaction were superior when the SCS therapies were started earlier ${ }^{8}$. In another report, the cost-effectiveness of the SCS was not found to be inferior compared to drug therapies ${ }^{13)}$.

\section{CONCLUSION}

In summary, the efficacy of spinal cord stimulation from 
post-zoster neuralgia represents an effective and safe method for post-zoster neuralgia on the spinal nerve. The benefits of SCS include minimal invasiveness that provides rapid relief of pain and improves quality of life.

\section{REFERENCES}

1. Asim SA, et al.: Post-Heretic Neuralgia. The Professional Medical Journal 23(01):56-59, 2016.

2. Bader MS: Herpes zoster: diagnostic, therapeutic, and preventive approaches. Postgraduate medicine 125(5):78-91, 2013

3. Bennett GJ and CPN Watson: Herpes zoster and postherpetic neuralgia: past, present and future. Pain Research and Management 14(4):275-282, 2009.

4. Cohen JI: Herpes zoster. New England Journal of Medicine 369(3):255-263, 2013

5. Gnann Jr, J and R Whitley: Clinical practice. Herpes zoster. The New England journal of medicine 347(5):340, 2002

6. Hanania MM and D Brietstein: Postherpetic neuralgia: a review. Cancer investigation 15(2):165-176, 1997
7. Harke H, et al.: Spinal cord stimulation in postherpetic neuralgia and in acute herpes zoster pain. Anesthesia \& Analgesia 94(3): 694-700, 2002

8. Iseki M, et al.: Efficacy of limited-duration spinal cord stimulation for subacute postherpetic neuralgia. Ann Acad Med Singapore 38(11):1004-1006, 2009

9. John AR and DH Canaday: Herpes zoster in the older adult. Infectious Disease Clinics 31(4):811-826, 2017

10. Kost RG and SE Straus: Postherpetic neuralgia-pathogenesis, treatment, and prevention. New England Journal of Medicine 335(1):32-42, 1996

11. Lojeski E and RA Stevens: Postherpetic neuralgia in the cancer patient. Current Review of Pain 4(3):219-226, 2000

12. Moriyama K: Effect of temporary spinal cord stimulation on postherpetic neuralgia in the thoracic nerve area. Neuromodulation: Technology at the Neural Interface 12(1): 39-43, 2009

13. Yanamoto $F$ and $K$ Murakawa: The effects of temporary spinal cord stimulation (or spinal nerve root stimulation) on the management of early postherpetic neuralgia from one to six months of its onset. Neuromodulation: Technology at the Neural Interface 15(2):151-154, 2012 